\title{
Quaderni
}

QUADERNI Communication, technologies, pouvoir

\section{Michel Foucault, la nouveauté comme différance de la philosophie}

Michel Foucault, Novelty as Philosophy's Differance

\section{Charles Ramond}

\section{(2) OpenEdition}

1 Journals

\section{Édition électronique}

URL : http://journals.openedition.org/quaderni/982

DOI : 10.4000/quaderni.982

ISSN : 2105-2956

\section{Éditeur}

Les éditions de la Maison des sciences de l'Homme

\section{Édition imprimée}

Date de publication : 5 mai 2016

Pagination : 67-79

\section{Référence électronique}

Charles Ramond, « Michel Foucault, la nouveauté comme différance de la philosophie », Quaderni [En ligne], 90 | Printemps 2016, mis en ligne le 05 mai 2018, consulté le 19 avril 2019. URL : http:// journals.openedition.org/quaderni/982; DOI : 10.4000/quaderni.982 


\section{$D$ ossier}

\section{Michel Foucault,}

la nouveauté

«Un cauchemar me poursuit depuis mon enfance: j'ai sous les yeux un texte que je ne peux pas lire, ou dont seule une infime partie m'est déchiffrable; je fais semblant de le lire, je sais que je l'invente; puis le texte soudain COMme se brouille entièrement, je ne peux plus rien lire ni même inventer, ma gorge se serre et je me réveille. »

\section{différance de} la philosophie

\section{Charles Ramond}

Université Paris 8 Vincennes Saint-Denis / Paris Lumière
Michel Foucault,

Dits et Écrits, 1967, n$^{\circ} 48$, t. 1, p. 623.

L'adjectif « nouveau », même dans une matière aussi ancienne que la philosophie, emporte avec lui une valorisation presque aussi irrésistible que la jeunesse. Comme les découvertes scientifiques, comme les créations artistiques, les systèmes philosophiques qui restent dans la mémoire de l'humanité, et la modifient, ont toujours eu quelque chose de nouveau, d'original. Nous savons tous, bien sûr, qu'il ne suffit pas de dire que quelque chose est " nouveau " pour dire quelque chose de nouveau. Que dire par exemple qu'au XVII ${ }^{e}$ siècle la pensée mécaniste apporte quelque chose de « radicalement nouveau » par rapport à l'aristotélisme ambiant, c'est énoncer quelque chose de bien connu, presque une banalité. Et qu'inversement, l'affirmation d'une continuité peut s'avérer originale, par exemple lorsque l'historien Zeev Sternhell a montré, contre l'opinion la plus généralement répandue, que l'idéologie de la révolution nationale n'était pas du tout une " nouveauté » en 1940, mais trouvait ses racines et sa nourriture dans les principaux courants de pensée français, à gauche comme à droite, sans interruption depuis la fin du XIX ${ }^{\mathrm{e}}$ siècle. Nous savons donc qu'on peut être banal et conventionnel en ayant sans cesse le mot «nouveau » à la bouche ou sous la plume, 
et original sans jamais le prononcer ou l'écrire. Malgré cela, le terme " nouveau » garde une grande force de séduction et d'attraction, comme si nous désirions obscurément glisser de la nouveauté de ce dont on nous parle à la nouveauté de ce que l'on nous dit.

L'œuvre de Michel Foucault ${ }^{1}$ est tout particulièrement exemplaire de ces paradoxes de la « nouveauté $»$. Foucault a été universellement reconnu comme un penseur original; et il est par ailleurs obsédé par la question de la «nouveauté ». Quel lien faire entre ces deux constatations ? Simple coïncidence, ou relation de cause à effet ? Question de grande conséquence pour la pensée contemporaine, tant Foucault y est lu, discuté, suivi, imité parfois. Mon hypothèse serait que la lecture attentive des textes de Foucault, jusque dans sa rhétorique et ses pratiques d'écriture, permet de mettre en évidence, chez lui, non seulement une attitude assez ambivalente vis-àvis de la notion de « nouveauté », mais même, et tout particulièrement à propos de cette notion de « nouveauté », une posture finalement plutôt traditionnelle en philosophie, ou du moins dans l'histoire de la pensée. Car Foucault, à plusieurs reprises, a déclaré «ne pas être philosophe $»^{2}$, et c'est une déclaration que nous n'avons aucune raison de considérer comme une coquetterie ou de ne pas prendre au sérieux. Comme le montrent ses violents débats avec Sartre et Derrida, quelque chose en Foucault fait obstacle, ou résiste, à l'entrée dans la philosophie contemporaine. Et ce quelque chose, pourrait bien être en lien direct, paradoxalement, avec la question de la « nouveauté ». On trouvera du moins, dans les pages qui suivent, quelques arguments en faveur de cette thèse.

\section{Le règne du « nouveau »}

À la lecture des ouvrages de Foucault, mais également des entretiens qu'il a donnés et des articles qu'il a pu écrire ${ }^{3}$, l'omniprésence de la notion de « nouveauté », et des adjectifs « nouveau » et « nouvelle», est particulièrement frappante. Il ne s'agit pas seulement d'un point de vue statistique, déjà considérable 4 , mais d'une caractérisation par Foucault de la philosophie contemporaine même, comme lorsqu'il déclare : "était philosophique au XIX $X^{e}$ siècle la réflexion qui s'interrogeait sur les conditions de possibilité des objets; est philosophie, aujourd'hui, toute activité qui fait apparaître un objet nouveau pour la connaissance ou la pratique - que cette activité relève des mathématiques, de la linguistique, de l'ethnologie ou de l'histoire » (DE 625), ou lorsqu'il caractérise par la « nouveauté » sa propre activité, sa propre démarche : «mon but sera de vous montrer comment les pratiques sociales peuvent en venir à engendrer des domaines de savoir qui non seulement font apparaître de nouveaux objets, de nouveaux concepts, de nouvelles techniques, mais aussi font naître des formes totalement nouvelles de sujets et de sujets de connaissance » (DE 1406-7).

Dès les premières pages de Surveiller et Punir, Foucault déclare que « l'objectif» de son livre est de proposer « une histoire corrélative de l'âme moderne et d'un nouveau pouvoir de juger» (SP 30). La caractérisation en termes de « nouveauté » n'est donc ni rare, ni marginale chez lui, mais touche qualitativement et quantitativement à son entreprise même. La présence de la « nouveauté », chez Foucault, ne se limite d'ailleurs pas à celle des termes « nouveau », « nouvelle», 
« nouveauté ». Elle se marque dans une série d'autres expressions, dans lesquelles elle est directement enveloppée, et qui la répandent presque partout, dans les textes et les entretiens. Par exemple, la scansion « jadis / maintenant» est assez fréquente : « Jadis, la déraison était mise hors jugement pour être livrée dans l'arbitraire aux pouvoirs de la raison. Maintenant, elle est jugée » (HF 522); ou encore « Jadis, le fou était libre pendant l'instant ténu où il se mettait à perdre sa liberté ; il est libre maintenant dans le large espace où il l'a déjà perdue » (HF 533)5.

L'opposition entre « jadis » et « maintenant» suppose bien évidemment l'apparition d'une « nouveauté », même si le terme lui-même est absent. Si le terme « innovation » est rare chez Foucault ${ }^{6}$, l'adverbe « désormais », souvent employé avec une certaine solennité par Foucault, est un autre marqueur de la notion de «nouveauté ». Qui dit que quelque chose est « désormais » fini signifie par là-même que quelque chose de « nouveau » est apparu, ou advenu. Par exemple : «Pendant tout le Moyen Âge, et longtemps au cours de la Renaissance, la folie avait été liée au Mal, mais sous la forme de transcendances imaginaires ; désormais, elle communique avec lui par les voies plus secrètes du choix individuel et de l'intention mauvaise » $(H F 152)^{7}$.

L'emploi fréquent et lourd de signification du terme « événement» participe également de cette omniprésence de la «nouveauté » chez Foucault, puisqu'un « événement » est comme le modèle de toute nouveauté. On lit ainsi : « il a bien fallu un événement fondamental - un des plus radicaux sans doute qui soit arrivé à la culture occidentale pour que se défasse la positivité du savoir classique et que se constitue une positivité dont nous ne sommes sans doute pas entièrement sortis» (MC 232); ou encore « Entre Montaigne et Descartes un événement s'est passé : quelque chose qui concerne l'avènement d'une ratio » $(\text { HF 58) })^{8}$.

Très souvent, l'adjectif « décisif » vient encore renforcer, s'il était nécessaire, la dimension de rupture, la dimension événementielle de l'événement lui-même, qui porte alors fréquemment, dans les textes de Foucault, le nom de « moment » : "Dans l'histoire de la déraison, "l'internement" désigne un événement décisif : le moment où la folie est perçue sur l'horizon social de la pauvreté » (HF 90) ${ }^{9}$.

Tout le chapitre 2 de la première partie de L'Archéologie du savoir (intitulé « les unités du discours ») est ainsi une réflexion sur la notion de « discontinuité », au cours de laquelle Foucault élabore la notion de « population d'événements » comme l'une des caractéristiques de « l'archéologie » que propose l'ouvrage : « Avant d'avoir affaire, en toute certitude, à une science, ou à des romans, ou à des discours politiques, ou à l'œuvre d'un auteur ou même à un livre, le matériau qu'on a à traiter dans sa neutralité première, c'est une population d'événements dans l'espace du discours en général » (AS 41).

Cette préoccupation constante de la nouveauté se marque ainsi à tous les niveaux des discours tenus par Foucault. On ne s'étonne donc pas qu'elle apparaisse aussi, sous la forme du recours à la notion de " génération », dans certains aperçus biographiques. De toute évidence, Foucault perçoit les 21 années (une génération) qui le 
séparent de Sartre comme une sorte d'abime dans la pensée, comme le montre le texte suivant, qui date de 1966 : «D’une façon très soudaine, et sans qu'il y ait apparemment de raison, on s'est aperçu, il y a environ 15 ans, qu'on était très, très loin de la génération précédente, de la génération de Sartre, de Merleau-Ponty - génération des Temps modernes qui avait été notre loi pour penser et notre modèle pour exister $\gg(D E 541) .{ }^{10}$

En quelques années, une pensée nouvelle peut surgir, qui relègue les précédentes au magasin des vieilleries. Foucault parle de $«$ mutation $»^{11}$ pour qualifier la « nouvelle histoire », et pour bien creuser l'écart et bien marquer la nouveauté apportée par sa "génération », va jusqu'à employer le vocabulaire religieux de la « conversion » : «je crois que, comme chez tous ceux de ma génération, s'est produit en moi, entre les années cinquante et soixante-cinq, une sorte de conversion » $(D E 629)^{12}$.

\section{Les nouveautés illusoires}

Foucault appelle "épistémè » un état général des savoirs et des pratiques de discours qui rend possible, à une époque donnée, un certain état des sciences et des pensées, et la présence simultanée de certaines thèses, certaines discussions, certaines opinions, et certaines controverses ${ }^{13}$. Le passage à une nouvelle épistémè modifie à ce point les manières de penser qu'il efface de la mémoire d'une époque, pour ainsi dire, la possibilité même du souvenir d'une époque régie par une autre épistémè ${ }^{14}$. La préoccupation constante de la « nouveauté » ne revient donc pas du tout, chez Foucault, à une valorisation du « progrès ». Bien loin d'être des étapes sur un chemin historique pourvu de sens, les nouvelles épistémès créent des mondes humains presque incommensurables, à la manière des différentes visions du monde proposées par les philosophies dans la Dianoématique de Martial Gueroult - si bien que, d'une certaine manière, la notion de « nouveauté » n'y a plus guère de pertinence, faute de comparaison possible et d'échelle commune.

De façon très frappante en effet, le recours à la notion d'épistémè permet à Foucault de retirer à la notion de « nouveauté » toute valeur ontologique ou épistémologique, au moment même où toute son écriture, comme nous venons de le voir, vibre d'attention pour le nouveau sous toutes ses formes.

Dans cette ambivalence à l'égard de la notion de « nouveauté », Foucault retrouve, me semble-til, un des gestes les plus classiques et les plus anciens de la philosophie, tel que Platon l'illustre dans la mythique « allégorie de la caverne » de La République. Le philosophe y est d'abord un voyant. Il voit ce que les autres hommes, enfermés dans l'obscurité de la caverne de leurs habitudes et de leurs préjugés, ne voient pas. Il voit la véritable source du monde, il voit les véritables découpages de la réalité, tandis que les autres se trompent en tout, confondant un petit feu avec le soleil, et leurs préjugés avec des vérités. Les paroles du philosophe, pour les hommes de la caverne, ont donc par définition quelque chose de « nouveau » et de choquant, en ce qu'elles remettent en cause la plupart des choses auxquels ils croient et tiennent. C'est la raison pour laquelle, nous dit Platon, le philosophe ne sera pas volontiers écouté. On se moquera de lui, et peut-être même on le mettra à mort. Mais quoi qu'il en soit 
du sort du philosophe, les vérités qu'il apporte ne sont « nouvelles » que pour ceux qui sont encore attachés à leurs anciennes croyances. En ellesmêmes, les vérités ne sont nullement destinées à rester « nouvelles ». Elles doivent au contraire se révéler, dans la confrontation avec la réalité, simplement vraies, c'est-à-dire vraies depuis toujours, sans aucune «nouveauté », tout comme un théorème de mathématiques n'est « nouveau » que pour ceux qui ne le connaissaient pas encore, mais éternel considéré en lui-même.

Et de fait, bien loin de se laisser déborder par la constante «nouveauté » de ce qu'il enregistre ou décrit ; bien loin d'en paraître choqué, ou troublé ; bien loin d'en conclure à l'inintelligibilité « des mots et des choses », Foucault développe au contraire imperturbablement, via les épistémès, une constante et complète légitimation conceptuelle de tous les phénomènes dont il traite, à la manière, disons, de Leibniz, le philosophe de l'âge classique dont il serait le plus proche par le geste philosophique général ${ }^{15}$ et par l'obsessionnelle critique de Descartes.

LES COMPOSSIBILITÉS. Du point de vue de la réalité des choses et des discours, une épistémè est d'abord un espace de compossibilités. Ce qui pouvait apparaître, aux hommes de la caverne, comme des contradictions insurmontables entre des thèses irréconciliables, est en réalité, replacé correctement dans l'épistémè de son époque, un ensemble de discours qui devaient avoir lieu simultanément, comme autant d'effets différents de causes plus profondes qu'eux. Les contradictions sont apparentes et superficielles, et disparaissent lorsqu'on se place au niveau de l'épistémè ou au niveau de l'archéologie.
Foucault y insiste un grand nombre de fois : «J'ai essayé de montrer dans Surveiller et Punir qu'il n'y a pas, à vrai dire, de contradiction entre le système apparemment archaïque des prisons et la criminologie moderne » (DE 1529).

Le « dire vrai » non contradictoire s'oppose donc à « l'apparence » contradictoire. La même thèse est souvent reprise dans Les Mots et les Choses: «il faut reconstituer le système général de pensée dont le réseau, en sa positivité, rend possible un jeu d'opinions simultanées et apparemment contradictoires » $(M C 89)^{16}$.

La distinction entre « l'apparent » et le « réel », entre « l'illusion » et la «vérité », fait système avec la distinction entre le « profond » et le « superficiel », ou encore avec le « contingent» et le « nécessaire », comme on peut le voir aussi dans Les Mots et les Choses : «Si on interroge le savoir qui a rendu possibles en même temps "ces opinions", on s'aperçoit que leur opposition est superficielle, et que si elle est nécessaire, c'est à partir d'une disposition unique qui ménage seulement, en un point déterminé, la fourche d'un choix indispensable » $(\text { MC 193 })^{17}$.

Dans le cadre d'une épistémè donnée, les oppositions d'opinions, les discussions, sont « superficielles », et leur compossibilité « profonde » pourvu qu'on sache les rapporter à « la fourche» antérieure d'un « choix indispensable » ou à la «profondeur archéologique» : «Envisagée dans $s a$ profondeur archéologique et non pas au niveau plus apparent des découvertes, des discussions, théories, ou options philosophiques, l'œuvre de Cuvier surplombe de loin ce qui allait devenir l'avenir de la biologie » (MC 287). 
LE PRINCIPE DE RAISON. Dans le cadre d'une épistémè, les discours apparemment contradictoires ne le sont pas. Ou mieux, leur caractère contradictoire est rendu nécessaire, et donc intelligible, par le cadre général que constitue l'épistémè ${ }^{18}$. On voit donc Foucault s'attacher sans relâche, sans défaillance, sans exception, à montrer que les épistémès qu'il décrit permettent de «comprendre » l'intégralité de ce qui s'y produit, si bien que le destin de toute « nouveauté » et par conséquent de tout « étonnement » y est de disparaître en tant que tels. Foucault reprend donc ici sans s'en écarter le chemin classique de la philosophie, qui va d'un « étonnement » premier suscité par une « nouveauté » apparente dans l'ordre des choses, à la disparition de cet étonnement (et donc de cette nouveauté) une fois que l'ordre des choses a été correctement décrit et compris. C'est ce qu'on appelle traditionnellement le « principe de raison », ou principium reddendae rationis, par lequel la tâche de toute pensée est conçue comme devant « rendre compte », ou « rendre raison $»$, du réel ${ }^{19}$.

Apparaissant avec régularité, les formulations en « on comprend » et en « on ne s'étonnera pas que » exposent cette intelligibilité et cette absence de surprise, que ce soit dans l'Histoire de la Folie: «ce que nous savons maintenant de la déraison nous permet de mieux comprendre ce qu'était l'internement » $(H F \text { 267 })^{20}$; «ne nous étonnons pas que la plus 'psychologique, des médications ait rencontré si vite son versant et ses confirmations organiques » (HF 360), dans la Volonté de Savoir : "cet épinglage du dispositif d'alliance et $d u$ dispositif de sexualité dans la forme de la famille permet de comprendre un certain nombre de faits : que la famille soit devenue depuis le XVIII ${ }^{e}$ siècle un lieu obligatoire d'affects, de sentiments, d'amour ; que la sexualité ait pour point privilégié l'éclosion de la famille; que pour cette raison elle naisse 'incestueuse'»(VS 143), et dans les autres ouvrages de Foucault ${ }^{21}$. Une fois que tout « est compris », ou compréhensible, ou expliqué, le « hasard » disparaît avec la « nouveauté ». On ne s'étonne donc pas, c'est le cas de le dire, de voir Foucault employer assez souvent la formule «ce n'est pas un hasard si » : «ce n'est pas un hasard si le sadisme [...] est né de l'internement [...]. Ce n'est pas un hasard non plus si toute la littérature fantastique [...] se situe [...] dans les hauts lieux de l'internement $»(\text { HF 381 })^{22}$.

LA PRÉVISION DU PASSÉ. L'intelligibilité intégrale du réel, dans le cadre d'une épistémè donnée, se manifeste enfin de façon particulièrement spectaculaire, dans les textes de Foucault, par l'adoption incessante de la «prévision du passé », c'est-à-dire de la description au futur d'événements que les hommes du passé ne connaissaient pas encore, mais que le narrateur du $\mathrm{XX}^{\mathrm{e}}$ siècle, qui sait ce qui a effectivement eu lieu dans les siècles qui ont suivi, peut annoncer. Par exemple : « ainsi s'est constitué à l'âge classique un espace d'empiricité qui n'avait pas existé jusqu'à la fin de la Renaissance, et qui sera voué à disparaître dès le début du XIX siècle » (MC 86).

Cette façon de narrer au futur des événements déjà passés est constante chez Foucault ${ }^{23}$. Du point de vue qui est ici le nôtre, elle est hautement significative. La simple prophétie se prononce au moment présent, se formule au futur, mais risque toujours d'être démentie par les faits. La prévision du passé (toujours assurée 
de réussir) ne peut donc être faite que d'un point de vue encore supérieur à celui d'un prophète ou d'un voyant, c'est-à-dire du point de vue d'un Dieu omniscient, pour lequel le déroulement des temps ne peut offrir aucune surprise, aucun événement, aucune véritable nouveauté. La prévision du passé signale ainsi, du point de vue de l'architectonique des systèmes philosophiques, un rationalisme intégral de type classique, très proche de ce qu' on peut trouver en effet dans de nombreux textes de Leibniz.

La prévision du passé est le temps des destinées, des annonces, des préfigurations et des promesses. Tout cela figure chez Foucault : «les réflexions scientifiques [...] maintiennent $[$...] une unité promise à se dissocier bientôt » (MC 253 : promesse) ; «à partir de ce déplacement de la question transcendantale, la pensée contemporaine ne pouvait éviter de ranimer le thème $d u$ cogito » (MC 334 : fatalité) ; «il viendra un jour où la profanation et toute sa gestuaire tragique n'aura plus que le sens pathologique de l'obsession » (HF 111 : annonce de la psychanalyse).

Le Neveu de Rameau est un lieu privilégié pour des « annonces » et des « préfigurations » : «Dernier personnage en qui folie et déraison se réunissent, le Neveu de Rameau est celui en qui le moment de la séparation est également préfiguré » (HF 364 ; id. 366) ; son délire « annonce Freud et Nietzsche » (HF 369) ; son rire " préfigure à l'avance et réduit tout le mouvement de l'anthropologie du XIX ${ }^{e}$ siècle ( HF 370) ; "quelque chose s'est passé, à l'époque même où Le Neveu de Rameau fut écrit, et qui promettait un changement décisif » (HF 373).
La prévision du passé s'accorde entièrement avec «l'archéologie», entendue comme recherche des conditions de possibilité ou de compossibilité de ce qui a été ou de ce qui a été dit, c'est-à-dire comme transcendantalisme objectif ${ }^{24}$. Celui qui connaît l'avenir, c'est-à-dire ce qui a eu lieu, sait en effet, en outre, ce qui aura été « possible». Le possible ne le surprendra pas plus que le réel : «à la connaissance qui devinait, au hasard [souligné $\mathrm{MF}$, des signes absolus et plus anciens qu'elle, s'est substitué un réseau de signes bâti pas à pas par la connaissance du probable. Hume est devenu possible » $(M C 74)^{25}$.

Les passages de l'Archéologie du savoir où Foucault récuse toute forme de transcendantalisme $^{26}$, et se moque de toute démarche préfigurative $^{27}$, ont donc certainement un côté dénégatif.

\section{Conclusion}

Son double geste simultané de mise en avant et de retrait de la « nouveauté » permet ainsi de révéler, chez Foucault, un imaginaire très classique et traditionnel de la philosophie. Comme chez Platon, la philosophie est d'abord une sorte de perception visuelle de la réalité. Elle a nécessairement un « objet ». Foucault déclare ainsi très significativement, comme une évidence première, qu' « il n'y a pas de discours philosophique sans objet $»^{28}$. À partir de là, la philosophie consiste à lutter contre les « mauvais découpages » de la réalité, contre les préjugés, les idées toutes faites (c'est par exemple tout le début de La Volonté de Savoir), et à leur substituer les « bons découpages » catégoriels, qui ne seront « nouveaux » que le temps de la disparition des préjugés. La philosophie se donne ainsi pour tâche première 
(c'est un leitmotiv chez Foucault) de « décrire » le monde, et de le « comprendre », jusqu'à en « prévoir » très exactement et complètement (quoique rétroactivement) le développement. Son interlocuteur est le monde, et pas les autres philosophes. L'idée que la philosophie pourrait consister à résoudre des problèmes plutôt qu'à décrire un objet n'affleure même pas. La suprématie dans la vision tient lieu de suprématie dans l'argumentation. D'où le cauchemar de perdre la vue, de ne plus pouvoir lire le monde. Dans une démarche qui est toute « positivité », le négatif des discussions réelles, avec de véritables interlocuteurs ayant émis et écrit de véritables objections, est ignoré. Foucault ne discute, dans la préface des Mots et des Choses, comme dans la conclusion de l'Archéologie du savoir, qu'avec lui-même, endossant l'habit d'un interlocuteur ou contradicteur fictif. Mais on ne trouve nulle part chez lui (sauf erreur) la reconnaissance, même momentanée, d'une impasse ou d'un échec, pas même d'une difficulté dans la pensée.

Le philosophe est un homme fort, un voyant, un prophète, presque un Dieu. La raison infaillible est de son côté, les émotions sont pour les femmes et pour les enfants, et les adversaires seront toujours ramenés à leurs émotions. Les tenants de la subjectivité dans l'histoire, nous l'avons vu, sont des « historiens en culottes courtes ». Les débats entre « le marxisme » et les « théories bourgeoises » ont beau «émouvoir quelques vagues et dessiner des rides à la surface : ce ne sont tempêtes qu'au bassin des enfants » (MC 274). Le philosophe ne reste pas «à la surface ». Ceux qui ne sont pas d'accord avec lui ne réfléchissent pas : ils « crient », ils «pleurent » (AS 25), ont «peur », ou «s'acharnent» (AS 284). L'archéologue n'est pas soumis à l'affectivité. $\mathrm{Il}$ « reste insensible à ce moment (émouvant $<i l>$ le veu $<t>$ bien) où pour la première fois, quelqu'un a été sûr d'une certaine vérité » (AS 196). Les «émotions» des «premières fois » sont bonnes sans doute pour les femmes. La « conscience », qui est l'ennemie, est «douce, muette, intime » (AS 285). Les arguments en faveur de la subjectivité ont été écrits par une "pauvre main », ils témoignent d'une « inquiétude », d'un « malaise», exprimés par des « murmures »; ils montrent la recherche d'une « tendre certitude, si consolante », d'une parole «fraîche»; ils témoignent d'une «frêle et incertaine existence », de gens qui ne « peuvent pas supporter » certains discours ( $A S$ 286). L'humanisme est toujours «mou» aux yeux de Foucault, ce qui est une façon de le dévaloriser, de lui faire subir, comme l'avait fait Sartre, une disqualification genrée ${ }^{29}$. La fixation sur une image plutôt traditionnelle du philosophe et de la philosophie, qui fait système avec tous les traits que nous avons relevés autour de la notion de « nouveauté », a ainsi peut-être paradoxalement retardé, « différé $»^{30}$, chez Foucault, une prise en compte plus exacte de ce qu'apportaient de véritablement nouveau, à son époque, les nombreux philosophes qui en étaient l'une des plus belles illustrations. 
$\mathrm{N} \cdot \mathrm{O} \cdot \mathrm{T} \cdot \mathrm{E} \cdot \mathrm{S}$

1. Je traiterai ici, pour des raisons de place et de quantité de travail nécessaire, de la première partie seulement de l'œuvre de Foucault, c'est-à-dire de la période correspondant au premier tome des Dits et Écrits, soit jusqu'en 1975. Les analyses et hypothèses présentées dans le présent article sont donc soumises à réfutation aussi par l'examen de la deuxième partie de l'œuvre. Abréviations utilisées : $D E$ pour Dits et Écrits 1954-1975, MC pour Les Mots et les Choses, HF pour Histoire de la folie à l'âge classique, SP pour Surveiller et Punir, AS pour L'Archéologie du Savoir, VS pour La Volonté de savoir. Sauf mention contraire, les soulignements sont les miens.

2. DE 466 : «je ne suis pas très philosophe»; ibid. 973 : «je ne me considère pas comme philosophe ».

3. Voir note 1 supra.

4. « Nouveau statut du discours médical », qui s'accompagne de «nouveaux critères », d'une « nouvelle découpe » de l'objet médical, d'un " nouveau mode d'enregistrement $d u$ discours médical », et d'un « nouveau fonctionnement » de celui-ci (DE $717 \mathrm{sq})$; Deleuze fait une «philosophie nouvelle » (DE 796 c'est un compliment de la part de Foucault; on sait que Deleuze et Foucault divergeront sur les «nouveaux philosophes »); au XVII ${ }^{\mathrm{e}}$ siècle " apparaissent des choses totalement nouvelles par rapport à la société féodale, à l'Empire carolingien et aux vieilles règles $d u$ droit romain »; «[...] un personnage totalement nouveau : le procureur; une notion absolument nouvelle : l'infraction » (DE 1447-8); « nouvelle définition du criminel » (DE 1458); au « grand savoir de l'enquête » succède un " nouveau savoir [...] de surveillance, d'examen » (DE 1465); «nouvelle forme matérielle de la richesse » (DE 1472); création d'un « nouveau et curieux type de pouvoir » (DE 1486); «cette nouvelle disposition entraîne l'apparition d'un nouveau problème » (MC 58) ; "l'épistémè de la culture occidentale [...] va prendre une configuration nouvelle» (MC 68); «à l'époque classique, [...] le langage a noué avec l'être de nouveaux rapports» (MC 109) ; " une nouvelle façon de nouer les choses à la fois au regard et au discours », " une nouvelle manière de faire l'histoire » (MC 143) ; « normes nouvelles » (HF 206); « espace nouveau dans lequel la folie, comme maladie, doit maintenant s'insérer » (HF 208) ; chez Diderot « les rapports de la raison et de la déraison prennent un visage tout nouveau» (HF 366) ; «cette acquisition d'une conscience temporelle de la folie [...] a nécessité l'élaboration de toute une série de concepts nouveaux » (HF 383-384); au $\mathrm{XIX}^{\mathrm{e}}$ siècle, « rapport nouveau à la folie » (HF 399); «structure nouvelle d'expérience 》 (HF 547); « enfermer : [...] une technologie nouvelle $\left(S P, 4^{\mathrm{e}}\right.$ de couv. $)$; « nouvelle théorie de la loi et du crime [...] ; nouvelle justification morale ou politique du droit de punir ; [...] pour la justice pénale, un âge nouveau» (SP 14); « nouvelle morale propre à l'acte de punir » (SP 19); « nouvelle stratégie pour l'exercice du pouvoir de châtier » (SP 97); « nouvelles formes d'accumulation du capital » (SP 103); « nouvelle législation criminelle, [...] nouvelle économie, $[\ldots]$ nouvelle technologie $d u$ pouvoir de punir ; [...] stratégie nouvelle » (SP 106); « nouvelle anatomie politique » (SP 162) ; « nouveau type de contrôle» (SP 167); «nouveau type de surveillance » (SP 205); « nouvelles mécaniques de pouvoir » au XVIII ${ }^{\mathrm{e}}$ siècle (SP 208) ; « nouveaux mécanismes de sanction normalisatrice $》(S P 216) ;$ «objectivités nouvelles » (SP 358); « nouveaux procédés de pouvoir » (VS 118-119) ; " nouveau dispositif [...] de sexualité» (VS 140); «nouvelles techniques pour maximaliser la vie» (VS 162) ; « histoire nouvelle» 
( $A S$ 19). Ce relevé, aussi significatif que possible, est loin d'être exhaustif.

5. Voir également SP 129 : « dans l'ancien système, le corps des condamnés devenait la chose du roi [...]. Maintenant, il sera plutôt bien social, objet d'une appropriation collective et utile $»$.

6. Je n'en ai trouvé que 3 occurrences dans tout le corpus que j'ai consulté : $D E 1352$ (dans le dialogue avec Chomsky); SP 222 : «Un 'pouvoir d'écriture'se constitue comme une pièce essentielle dans les rouages de la discipline. Sur bien des points, il se modèle sur les méthodes traditionnelles de la documentation administrative. Mais avec des techniques particulières et des innovations importantes » (le mot « innovation» est répété à la dernière ligne de la page); et VS 156 : «la médecine des perversions et les programmes de l'eugénisme ont été, dans la technologie du sexe, les deux grandes innovations de la seconde moitié du $X I X^{e}$ siècle ».

7. Autres exemples : MC $53:$ : le langage a désormais pour nature première d'être écrit »; MC 65 : « désormais les belles figures rigoureuses et contraignantes de la similitude vont être oubliées»; MC 230 : «alors que » auparavant / « désormais »; HF 70 : « désormais la misère n'est plus prise dans une dialectique de l'humiliation et de la gloire »; HF 103 : « désormais, de nouveaux rapports sont instaurés entre l'amour et la déraison »; HF 111 : « désormais, il y aura le système dénoncé de l'extérieur comme ensemble illusoire»; HF 173 : «pour le christianisme de la Renaissance, [...] folie de l'incarnation d'un dieu fait homme ; [...] désormais <sc. "pour le classicisme »>, la folie indique l'animalité»; HF 207 : «jadis, la maladie était permise par Dieu. [...] désormais elle se trouvera tout investie de sagesse divine $»$. Ici comme ailleurs, la liste n'est nullement exhaustive.

8. Voir également $D E 709,732,734,1094,1125,1135$, 1144, 1151, 1163 ; MC 229 : « événement radical »;
HF 220 : «à la fin du XVII siècle, un événement s'est produit [...] : la définition des troubles qu'on appelle d'abord 'vapeurs', et qui prendront une si grande extension au XVIII' siècle sous le nom de 'maladies de nerfs'”.

9. Voir aussi MC 32 : «il faut nous arrêter un peu en ce moment $d u$ temps où la ressemblance va dénouer son appartenance au savoir et disparaître, au moins pour une part, de l'horizon de la connaissance ».

10. Voir DE 546 : «les gens de notre génération».

11. DE 613 (1967) : « certains ignorent la très importante mutation du savoir historique en ouvre depuis déjà plus de vingt ans » (Foucault met ici en regard les ouvrages de Braudel, Furet, Leroy-Ladurie, avec ceux de Dumézil, de Lévi-Strauss et de Lacan).

12. Voir aussi HF 382 : «toute cette brusque conversion de la mémoire occidentale, à la fin du XVIII siècle». 13. Voir AS 260 : «l'épistémè, ce n'est pas ce qu'on peut savoir à une époque, compte tenu des insuffisances techniques, des habitudes mentales, ou des bornes posées par la tradition ; c'est ce qui, dans la positivité des pratiques discursives, rend possible l'existence des figures épistémologiques et des sciences ».

14. MC 88-89 : « De là aussi le fait qu'une pareille disposition ait disparu au point que les âges ultérieurs ont perdu jusqu'à la mémoire de son existence : c'est qu'après la critique kantienne, et tout ce qui s'est passé dans la culture occidentale à la fin du XVIII siècle, un partage d'un nouveau type s'est instauré».

15. On lit en $A S 72$, à propos des « discours des médecins, au XIX ${ }^{\mathrm{e}}$ siècle » : "Des uns aux autres quel enchaînement, quelle nécessité ? Pourquoi ceux-ci, et pas d'autres? Il faudrait trouver la loi de toutes ces énonciations diverses, et le lieu d'où elles viennent ». Même déclaration typiquement leibnizienne en $A S 79$ : « ne pourrait-on pas trouver une loi qui rende compte de l'émergence successive ou simultanée de concepts disparates $» ?$ 
16. Voir aussi MC 135 : «non pas calculer le dénominateur commun des opinions, mais définir à partir de quoi il était possible qu'il y eût des opinions - telles ou telles - sur le langage ». Pourvu que l'on découvre le bon point de vue, toutes les « opinions » («telles ou telles » opinions) se révèlent « possibles » simultanément, c'est-à-dire compossibles.

17. Tout MC 251 développe l'opposition entre les « mouvements de surface » et le «fondamental » où les connaissances « $s$ 'enracinent dans leur positivité». 18. Voir HF 533 : «C'est pourquoi, dans l'expérience classique, la folie peut être en même temps un peu criminelle, un peu feinte, un peu immorale, un peu raisonnable aussi [souligné $\mathrm{MF}$ ]. Ce n'est pas là une confusion dans la pensée, ou un moindre degré d'élaboration; ce n'est que l'effet logique d'une très cohérente structure. »

19. Foucault formule lui-même le «principe de raison » en AS 42 : «la description des événements du discours pose une tout autre question : comment se fait-il que tel énoncé soit apparu et nul autre à sa place ?»; Ibid. : «on doit montrer pourquoi <un énoncé> ne pouvait être autre qu'il était ».

20. Voir HF 264 : « on comprend que le héros tragique [...] ne puisse jamais être fou »; HF 354 : « on peut comprendre pourquoi la folie comme telle a disparu du théâtre à la fin du XVII siècle pour ne reparaître guère avant les dernières années du siècle suivant». 21. Voir par exemple SP 60 : « on peut comprendre à partir de là certains caractères de la liturgie des supplices»; SP 216 : « On comprend que le pouvoir de la norme fonctionne facilement à l'intérieur d'un système de l'égalité formelle, puisque », etc. ; SP 264 : "Le supplice achève logiquement une procédure commandée par l'Inquisition. La mise en 'observation' prolonge naturellement une justice envahie par les méthodes disciplinaires et les procédures d'examen. Que la prison cellulaire [...] soit devenue l'instrument moderne de la pénalité, quoi d'étonnant? Quoi d'étonnant si la prison ressemble aux usines, aux écoles, aux casernes, aux hôpitaux, qui tous ressemblent aux prisons? » : si tout est « logique » et « naturel », il n’y a en effet rien « d'étonnant »; SP 298 : « on comprend que la justice ait adopté si facilement une prison qui n'avait point pourtant été la fille de ses pensées. Elle lui devait bien cette reconnaissance. »; VS 191 : «Sur ce fond peut se comprendre l'importance prise par le sexe comme enjeu politique. C'est qu'il est à la charnière des deux axes le long desquels s'est développée toute la technologie politique de la vie (disciplines du corps / régulation des populations) ».

22. Voir également HF 401 : « une telle rencontre n'est sans doute pas due au hasard; et pourtant, entre les spécifications que propose Sauvages, et les catégories qui sont indiquées sur les registres de Charenton ou de Saint-Lazare, il n'y a pratiquement aucun point commun »-donc " pas de hasard » à ce que se « rencontrent» des notions qui n'ont pourtant «pratiquement aucun point commun»; HF 481 : «ce n'est pas un hasard [...] si le XIX siècle a demandé d'abord à la pathologie de la mémoire, de la volonté et de la personne, ce qu'était la vérité du souvenir, du vouloir et de l'individu»; HF 482 : «l'éventualité pour l'homme d'être fou et la possibilité d'être objet se sont rejointes à la fin du XVIII siècle, et cette rencontre a donné naissance à la fois (11 n'y a pas, en ce cas, de hasard de date) aux postulats de la psychiatrie positive et aux thèmes d'une science objective de l'homme» - mais Foucault n'évoque jamais de « hasard de date», tout au contraire.

23. MC 111, §2: "parvenue à ce point de généralité, la fonction du verbe n'aura plus qu'à se dissocier, dès que disparaîtra le domaine unitaire de la grammaire générale. Lorsque la dimension du grammatical sera libérée, la proposition ne sera plus qu'une unité de syntaxe. Le verbe y figurera parmi les autres mots 
avec son système propre de concordances, de flexions et de régime. Et à l'autre extrême, le pouvoir de manifestation du langage réapparaîtra dans une question autonome, plus archaïque que la grammaire. Et pendant tout le XIXe siècle, le langage sera interrogé dans sa nature énigmatique de verbe [...]. De Hegel à Mallarmé, cet étonnement devant les rapports de l'être et du langage, balancera la réintroduction $d u$ verbe dans l'ordre homogène des fonctions grammaticales »; MC 143 : «le XIX siècle retrouvera, après ce pur tableau des choses, la possibilité renouvelée de parler sur des mots »; MC 144 : «et c'est dans ce temps classé [...] que les historiens du XIX siècle entreprendront d'écrire une histoire enfin 'vraie'»; MC 238 : «à partir de Smith le temps de l'économie ne sera plus celui de [...] ; ce sera le temps intérieur d'une organisation»; HF 38 : «l'élément 'tragique, et l'élément 'critique' iront désormais en se séparant toujours davantage, ouvrant dans l'unité profonde de la folie une béance qui ne sera plus jamais recouverte »; HF 149 : "lorsque le XIX siècle décidera de faire passer à l'hôpital l'homme de déraison, et lorsqu'il fera en même temps de l'internement un acte thérapeutique [...], il le fera par un coup de force» HF 493 : « et derrière toutes ces images, un mythe commence à prendre figure, qui sera une des grandes formes organisatrices de la psychiatrie au XIX $X^{e}$ siècle, le mythe des trois Natures : Nature-Vérité, Nature-Raison, et Nature-Santé »; SP 121 : « il faudra attendre longtemps pour que l'homo criminalis devienne un objet défini dans un champ de connaissance ». Cette liste ne donne qu'un très faible aperçu des occurrences de ce procédé d'écriture constant chez Foucault.

24. MC 13 : «on voudrait mettre au jour le champ épistémologique, l'épistémè où les connaissances [...] enfoncent leur positivité et manifestent ainsi $<l$ '> histoire de leurs conditions de possibilité »; MC 68 : « essayer de restituer les modification qui ont altéré le savoir lui-même, à ce niveau archaïque qui rend possibles les connaissances et le mode d'être de ce qui est à savoir »; MC 46: «le savoir du XVI siècle à son niveau archéologique - c'est-à-dire dans ce qui l'a rendu possible»; MC 145 : « ce champ [...] définit la condition de possibilité de l'histoire naturelle»; HF 225 : «nous tenterons de ressaisir le mouvement par lequel est devenu $<\mathrm{sic}>$ possible [souligné $\mathrm{MF}$ ] une connaissance de la folie ».

25. Cf. MC 77 : un « réseau unique de nécessités» a « rendu possibles ces individualités que nous appelons Hobbes, ou Berkeley, ou Hume, ou Condillac».

26. AS 275 : «il m'a paru que là, pour l'instant, était l'essentiel : affranchir l'histoire de la pensée de sa sujétion transcendantale » (Kant est cité p. 277). Foucault a certainement affranchi l'histoire du « sujet» transcendantal, mais sa recherche constante n'en reste pas moins transcendantale au sens premier de « recherche des conditions de possibilité».

27. AS 195 : "Chercher dans le grand amoncellement $d u$ déjà-dit le texte qui ressemble 'par avance' à un texte ultérieur, fureter pour retrouver, à travers l'histoire, le jeu des anticipations ou des échos, [...] ce sont des amusements sympathiques d'historiens en culottes courtes $»$.

28. DE 1390.

29. Il n'y a même pas, à ses yeux, d'humanisme « dur », qui serait plus acceptable : "à bien y réfléchir, je dirais que 'humanisme mou' est une formule purement redondante, et que 'humanisme'implique de toute manière 'mollesse'» (DE 643). Voir aussi ibid. 705 , où Foucault explique qu'il faut « mettre entre $p a-$ renthèses toutes les vieilles formes de continuité molle par lesquelles on atténue d'ordinaire le fait sauvage $d u$ changement ». Le domestique est « mou », pas le « sauvage » : thème classique de la décadence par / dans le féminin, de Platon à Rousseau.

30. C'est pour cela, le lecteur l'aura compris, que nous 
avons intitulé ce texte, de façon toute derridienne, « la nouveauté comme différance de la philosophie».

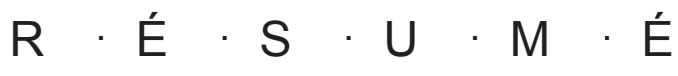

La notion de « nouveauté » est omniprésente dans les textes de Foucault. Mais d'un autre côté, sa construction de la notion d'épistémè le reconduit à un rationalisme intégral de type classique, dans lequel la philosophie a pour tâche de " comprendre » le « monde », et de mettre fin (comme illusoire) à toute nouveauté comme à tout étonnement. Nous montrons la cohérence de ce double geste, et à travers cela l'attachement de Foucault à une figure classique de la philosophie, rationnelle et virile, qui l'empêche peut-être, dans une certaine mesure, d'en apprécier les accomplissements les plus contemporains.

\section{Abstract}

Michel Foucault, Novelty as Philosophy's Differance

The notion of «novelty» (nouveauté) is omnipresent in Foucault's work. On the other hand, his construction of the notion of «épistémè» leads him back to an integral rationalism of the classical type, in which philosophy is meant to «understand» the «world» and put an end (as illusory) to all novelty as well to as any event. We inspect the coherence of this double-gesture, and beyond that Foucault's attachment to a classical figure of philosophy, as rational and virile, which prevented him perhaps, to a certain degree, from appreciating its more contemporary accomplishments. 
